\title{
Esculetin Downregulates the Expression of AML1-ETO and C-Kit in Kasumi-1 Cell Line by Decreasing Half-Life of mRNA
}

\author{
Sharad Sawney, ${ }^{1,2}$ Rashi Arora, ${ }^{2}$ Kamal K. Aggarwal, ${ }^{1}$ and Daman Saluja ${ }^{2}$ \\ ${ }^{1}$ University School of Biotechnology, Guru Gobind Singh Indraprastha University, Sector 16-C, Dwarka, New Delhi 110078, India \\ ${ }^{2}$ Dr. B.R. Ambedkar Center for Biomedical Research University of Delhi, Delhi 110007, India \\ Correspondence should be addressed to Daman Saluja; dsalujach59@gmail.com
}

Received 30 December 2014; Accepted 23 February 2015

Academic Editor: Akira Hara

Copyright (c) 2015 Sharad Sawney et al. This is an open access article distributed under the Creative Commons Attribution License, which permits unrestricted use, distribution, and reproduction in any medium, provided the original work is properly cited.

\begin{abstract}
One of the most frequent genetic aberrations in acute myeloid leukemia (AML) is chromosomal translocation between $A M L 1 / R U N X 1$ on chromosome 21 and ETO gene on chromosome 8 resulting in the expression of chimeric oncogene AML1-ETO. Although patients with $\mathrm{t}(8 ; 21)$ translocation have good prognosis, 5 -year survival is observed only in $50 \%$ of the cases. AML1ETO translocation is usually accompanied by overexpression of mutant $C$-Kit, a tyrosine kinase, which contributes to uncontrolled proliferation of premature blood cells leading to relapse and poor prognosis. We illustrate the potential use of esculetin on leukemic cell line, Kasumi-1, bearing t(8;21) translocation and mutated C-Kit gene. Esculetin decreases the expression of AML1-ETO at both protein and transcript level within 24 hours of treatment. Half-life of AML1-ETO mRNA was reduced from 7 hours to 1.5 hours. Similarly half-life of C-Kit mRNA was reduced to 2 hours from 5 hours in esculetin treated cells. Esculetin also perturbed the expression of ectopically expressed AML1-ETO in U937 cells. The decreased expression of AML1-ETO chimeric gene was associated with increased expression of LAT1 and RUNX3 genes, targets of AML1. We envisage that discovery of a drug candidate which could target both these mutated genes would be a considerable breakthrough for future application.
\end{abstract}

\section{Introduction}

The World Health Organization reported 14.1 million new cases and 8.2 million cancer deaths worldwide in 2012. It is prognosticated that, over the next few years, cancer including leukemia will surpass cardiovascular diseases [1]. Based on the clinical onset and lineage of the transformed immature leukocytes, leukemia can be acute or chronic and myeloid or lymphoid in origin. Chromosomal abnormalities, translocations, gene mutations, deregulation of gene expression, and epigenetic transmutations in the hematopoietic stem cells are the major contributory factors that interfere with the hematopoietic differentiation and result in the development of leukemia. Acute myeloid leukemia (AML) is a major class of leukemia, accounting for $20 \%$ of acute leukemia, wherein role of translocations and mutations in the malignancy of hematopoietic system is well established [2]. These genetic alterations broadly fall in two categories, Class I and Class II.

Class I is comprised of mutations in genes such as tyrosine kinases (KIT and FLT3 genes) that alter signal transduction pathways and thereby increase cellular proliferation and/or survival of hematopoietic progenitor cells [3]. Mutations and alterations in transcription factors, such as AML1 (RUNX1), CEBPA, and MLL, resulting in the disruption of cellular differentiation come under Class II mutation. Class II mutations, though the first hit, are insufficient to cause leukemia and require secondary hit in genes of Class I for the phenotype [4]. Most prevalent Class II mutations under AML are translocation between RUNX1 $(C B F \alpha)$ gene on chromosome 21, ETO (or MTG8) gene on the 8 chromosome $\mathrm{t}(8 ; 21)$ (q22;q22) [5], and inversion inv16 (CBF $\beta-M Y H 11)$ (p13q22) [6]. The $t(8 ; 21)$ results in a chimeric oncogene AML1-ETO, resulting in expression of a fused oncoprotein AML1-ETO (AE), and is found in $20 \%$ of the AML patients [2, 7]. The conserved Runt Homology Domain (RHD) required for DNA binding is at the N terminal of AML1 protein, whereas the transactivation and repressor domains span the $\mathrm{C}$ terminal region of the protein [1]. AML1 gene acts as a transcription activator for majority of hematopoietic genes like T and B-cell receptors, PU1, GATA, interleukin-3 
(IL-3), macrophage colony stimulating factor receptor $(M-$ CSFR), and so forth [8]. The ETO protein, a corepressor, is characterized by its four conserved Nervy Homology Regions (NHR1 to NHR4) that play an important role in proteinprotein interactions [9]. The chimeric AML1-ETO protein is comprised of $\mathrm{N}$ terminal DNA binding domain of $A M L 1$ while the $A M L 1$ activation domains are replaced with almost the entire ETO protein. As a result, the AE chimeric protein inhibits the expression of AML1 target genes instead of activating them through recruitment of corepressors and HDACs [10]. Interactions within the family of ETO proteins result in the formation of high molecular weight oligomers, further increasing the association of AE protein with $\mathrm{N}-\mathrm{CoR}$, SMRT, and other corepressors. These interactions are indispensable for the functioning of the chimeric AML1-ETO oncoprotein $[9,11]$.

The AML1-ETO repressor protein actively competes with AML1 and suppresses the transcription of $A M L 1$ target genes such as E-proteins, PU.1, C/EBP $\alpha$, and GATA-1 transcription factors that play a vital role in hematopoiesis $[2,10]$. Genes of DNA repair pathway are also inhibited by $\mathrm{AE}$ protein, resulting in the uninterrupted expansion of the mutated diseased cells [12].

The mutations in AML1 mark the genesis of leukemia; however, accumulation of secondary mutations are necessary for the manifestation of aggressive forms of acute leukemia [13]. About $70 \%$ of AML cases witness overexpression of receptor tyrosine kinase KIT. Though mutations in KIT gene are sporadic (as low as $~ 5 \%-6 \%$ ) [14, 15], its incidence increases to as high as $30 \%$ in patients (37\% of adult cases and $19 \%$ of paediatric cases) with $\mathrm{AE}$ translocation and is associated with poor prognosis $[3,16]$.

The current treatment of AML1-ETO acute myeloid leukemia involves the use of cytarabine (Ara-C) and anthracycline (such as daunorubicin). These drugs often result in complete remission in 60 to $80 \%$ of patients with a median survival less than 2 years and an overall survival of about 5 years [17]. Therefore, identification of novel therapeutic targets and drugs for treatment of $t(8 ; 21)$ positive AML is necessary for better patient management. The discovery of imatinib (Gleevec), an inhibitor of tyrosine kinase activity of $\mathrm{BCR} / \mathrm{ABL}$, was a breakthrough in the treatment of chronic myeloid leukemia (CML). It has the potential to block the activity of mutated KIT and PDGFR and has proven to be a promising candidate for the treatment of CML. Along similar lines, attempts are being made to modulate the levels of $\mathrm{AE}$ oncoprotein in $\mathrm{t}(8 ; 21)$ positive AML patients. Calpain $\mathrm{B}$ inhibitors have been shown to trigger AE protein degradation and decrease cell viability and clonogenic potential in Kasumi-1 cells [18].

The ectopic expression of Cathepsin G, a serine protease, induces degradation of AE protein in Kasumi-1 cells [19]. Similarly, corticosteroids were also shown to trigger ubiquitin mediated proteasomal degradation of $\mathrm{AE}$ chimeric protein [20]. Exploring the effect of natural compounds from traditionally known medicinal plants has attracted increasing attention in recent years. Oridonin, a diterpenoid, isolated from Isodon rubescens, was shown to cleave AML1-ETO protein in caspase-3 dependent manner [21].
Esculetin, a natural coumarin found in several plants including Artemisia capillaris, leaves of Citrus limonia [22], and Ceratostigma willmottianum [23], has been shown to protect against $\mathrm{N}$-methyl-N-nitrosourea-induced mammary carcinogenesis in rodents [24]. It inhibits proliferation of human breast cancer cells [25] and induces apoptosis in human leukemic HL-60 cell line [26]. Esculetin is, therefore, magnetizing the focus of active research as a potential anticancer drug. We tested whether esculetin can categorically target AML1-ETO so that it could be used in treating $t(8 ; 21)$ AML. Since C-Kit mutations are highly prevalent in AML1ETO patients (about 30\% cases), we also compared the levels of C-Kit in the presence and absence of esculetin using Kasumi-1 cell line as it possesses both AML1-ETO and CKit activating mutation N822K. Our results illustrate that esculetin has the antiproliferating activity against Kasumi1 cell line. Our results evince that esculetin decreased the levels of both C-Kit protein and AML1-ETO chimeric protein. Furthermore, the expression of both the genes is reduced drastically at the transcript level due to enhanced degradation of the AML1-ETO and C-Kit mRNA.

\section{Materials and Methods}

2.1. Materials. Esculetin (6,7-dihydroxycoumarin, $98 \%$ purity) was purchased from Sigma-Aldrich and dissolved in dimethyl sulfoxide (DMSO, vehicle). RPMI 1640 and all other chemicals, not specifically cited here, were purchased from Sigma-Aldrich. Fetal bovine serum (FBS) was purchased from GIBCO-BRL (South America Origin). An enhanced chemiluminescence (ECL) kit was purchased from Pierce. Antibody against AML1-ETO chimeric protein was purchased from Diagenode (C15410080) (Belgium). Antibody against $\mathrm{C}$-Kit and $\beta$-actin was purchased from Cell Signaling (USA). Peroxidase-labeled donkey anti-rabbit and antimouse immunoglobulin was purchased from Santa Cruz (USA).

2.2. Leukemia Cell Lines. Kasumi-1, a human leukemia cell line (M2 AML), was a kind gift from Professor Olef Hendrich (Newcastle University, Northern Institute for Cancer Research, UK). U937 and U937AE cell lines were a kind gift from Professor Dong-Er Zhang (Department of Pathology \& Division of Biological Sciences Moores, UCSD Cancer Center, University of California, San Diego, USA). Kasumi1 and U937 were grown in RPMI-1640 (Sigma) enriched with $15 \%$ fetal bovine serum (Gibco, South American Origin) at $37^{\circ} \mathrm{C}$ under $5 \% \mathrm{CO}_{2}$ atmosphere. U937 and U937AE were maintained as described by Burel et al. [27]. Culture media were changed periodically to ensure the cellular integrity.

2.3. RNA Isolation and Real Time PCR. For gene expression studies at mRNA level, Kasumi-1 cells were treated with $100 \mu \mathrm{M}$ esculetin for 24 hours and 48 hours. To determine the half-life of AML1-ETO and C-Kit mRNA, Kasumi-1 cells treated with $100 \mu \mathrm{M}$ esculetin for 24 hours were subsequently treated with $50 \mu \mathrm{M}$ of $\alpha$-amanitin for different time intervals [28]. A control set without esculetin treatment was analysed 
TABLE 1

\begin{tabular}{lccc}
\hline Primer name & Forward primers & Reverse primer & Product size (bp) \\
\hline GUSB & CTCATTTGGAATTTTGCCGATT & CCGAGTGAAGATCCCCTTTTTA & 80 \\
$18 S$ r RNAS & GTGGTGTTGAGGAAAGCAGACA & TGATCACACGTTCCACCTCATC & 79 \\
AML1-ETO & CTACCGCAGCCATGAAGAACC & AGAGGAAGGCCCATTGCTGAA & 395 \\
$C-$ Kit & CACCGAAGGAGGCACTTACAC & GGAATCCTGCTGCCACACA & 148 \\
LAT1 & GAGCTACGAGAACGAGGGTG & GCCTGGGTTGTGATAGTCGT & 157 \\
RUNX3 & GGATGGTACGGTGGTGACTG & CTTGATGGCTCGGTGGTAGG & 208 \\
\hline
\end{tabular}

in parallel. The expression of mRNA of AML1-ETO was also compared in U937AE cell line by culturing the cells in absence of tetracycline for 24 hours, to induce transcription of AML1-ETO. Thereafter cells were incubated with or without esculetin for 48 hours. Total RNA was isolated using RNA easy kit (Qiagen) as per manufacturer's protocol. RNA concentration was determined using Nanodrop (Thermo) and the quality and integrity were checked on $1 \%$ agarose gel. First strand cDNA was synthesized using $1 \mu \mathrm{g}$ of total RNA in $20 \mu \mathrm{L}$ reverse transcriptase reaction mixture according to the manufacturer's protocol (Thermo). Semiquantitative PCR was carried out typically in $20 \mu \mathrm{L}$ PCR reaction mixture using primers specific for AML1-ETO and C-Kit (5 pmol each) and $1 / 10$ volume of cDNA preparation $(1 \mu \mathrm{L})$ as template, PCR buffer with $\mathrm{MgCl}_{2}, 2 \mathrm{mM}$ dNTPs, (final concentration $0.2 \mathrm{mM}$ ) and Taq polymerase (1 unit). Initial denaturation was performed at $95^{\circ} \mathrm{C}$ followed by 28 cycles of denaturation at $95^{\circ} \mathrm{C}$ for 30 seconds, annealing at $60^{\circ} \mathrm{C}$ for $30 \mathrm{sec}$, and extension at $72^{\circ} \mathrm{C}$ for $30 \mathrm{sec}$. Amplicons were electrophoresed on $1-2 \%$ agarose gel. For quantitative PCR, 1X SYBR Green dye mix (Roche), 2 pmoles of each primer, and 1/20 volume of cDNA was used in $20 \mu \mathrm{L}$ reaction on the BIO-RAD iCycler iQ system (BioRad, Hercules, CA, USA). The fluorescence threshold value was calculated using the iCycle iQ system software. Copy number for a given RNA was calculated using standard curve of known template concentration. Standard curve was made by plotting fluorescence obtained in qPCR against the serially diluted known concentration of plasmid having AML1-ETO and C-Kit DNA fragments used for qPCR. 18S rRNAs and GUSB were taken as internal control [29] (see Table 1).

2.4. Preparation of Total Cell Extracts and Immunoblot Anal$y$ sis. After the desired period of incubation with esculetin (100 $\mu \mathrm{M}$ for $12-48$ hours), cells were washed with PBS and resuspended in RIPA lysis buffer. The mixture was centrifuged for $15 \mathrm{~min}(10,000 \mathrm{~g})$ after mixing for $30 \mathrm{~min}$ at $4^{\circ} \mathrm{C}$. Protein concentration in the supernatant (whole cell lysate) was determined using BCA protein estimation kit (Bangalore Genei) and equal amount of protein of control and esculetin treated sample $(100 \mu \mathrm{M})$ was resolved on $8 \%$ SDSPAGE and transferred onto PVDF membrane. The Western blot was probed for the desired proteins and detected using ECL kit (Pierce) followed by exposure on luminescent image analyzer (Fujifilm LAS-4000).

2.5. Statistical Analysis. Unless otherwise indicated, q-PCR and Western blot analysis were repeated at least thrice. The

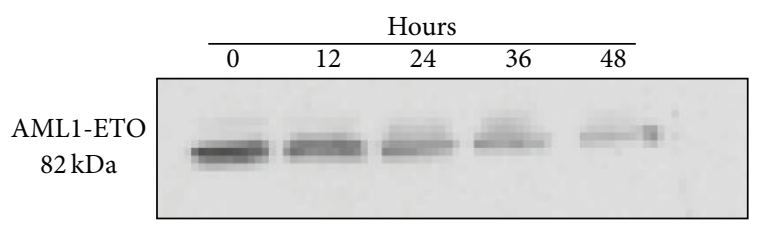

(a)

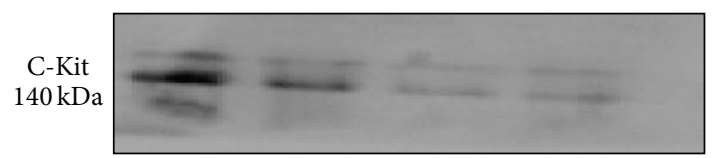

(b)

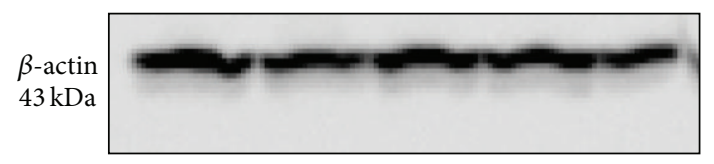

(c)

FIGURE 1: Esculetin treatment downregulates the expression of AML1-ETO chimeric protein and C-Kit protein in Kausmi-1 cell line. Whole cell lysates from Kausmi-1 cells treated with $100 \mu \mathrm{M}$ of esculetin for the indicated periods of time (in hours) were analysed. Equal amount of protein is loaded in each lane of the denaturing gel and immunoblotted with anti-AML1-ETO (a), anti-C-Kit (b), and anti- $\beta$-actin antibodies (c); $\beta$-actin was used as the loading control.

results of various parameters were analysed using Graph Pad Prism 6.0. All the data was expressed as mean \pm SD. The statistical significance was assessed by one way and two way analyses of variance followed by Bonferroni's multiple comparisons test with a confidence level of $(P<0.05)$.

\section{Results and Discussion}

Progression of acute leukemia is associated with the partial loss in the function of homeostasis of hematopoietic stem cells [30]. Acute myeloid leukemia patients harbouring the $t(8 ; 21)$ translocation are generally given a good prognosis at the initiation of treatment and absolute remission is achieved in most of the cases. Dismally, the disease recurs in $30-40 \%$ of these patients with overall survival being 5 years in less than $50 \%$ of the patients [31]. Higher relapse rate and shorter life span are witnessed in cases with high expression of $\mathrm{C}$-Kit mutations [32]. As in the case of U937 and HL-60 cell lines $[26,33]$, we observed concentration and time dependent antiproliferating activity of esculetin against Kasumi-1 cell line, which possess both AML1-ETO t $(8: 21)$ 

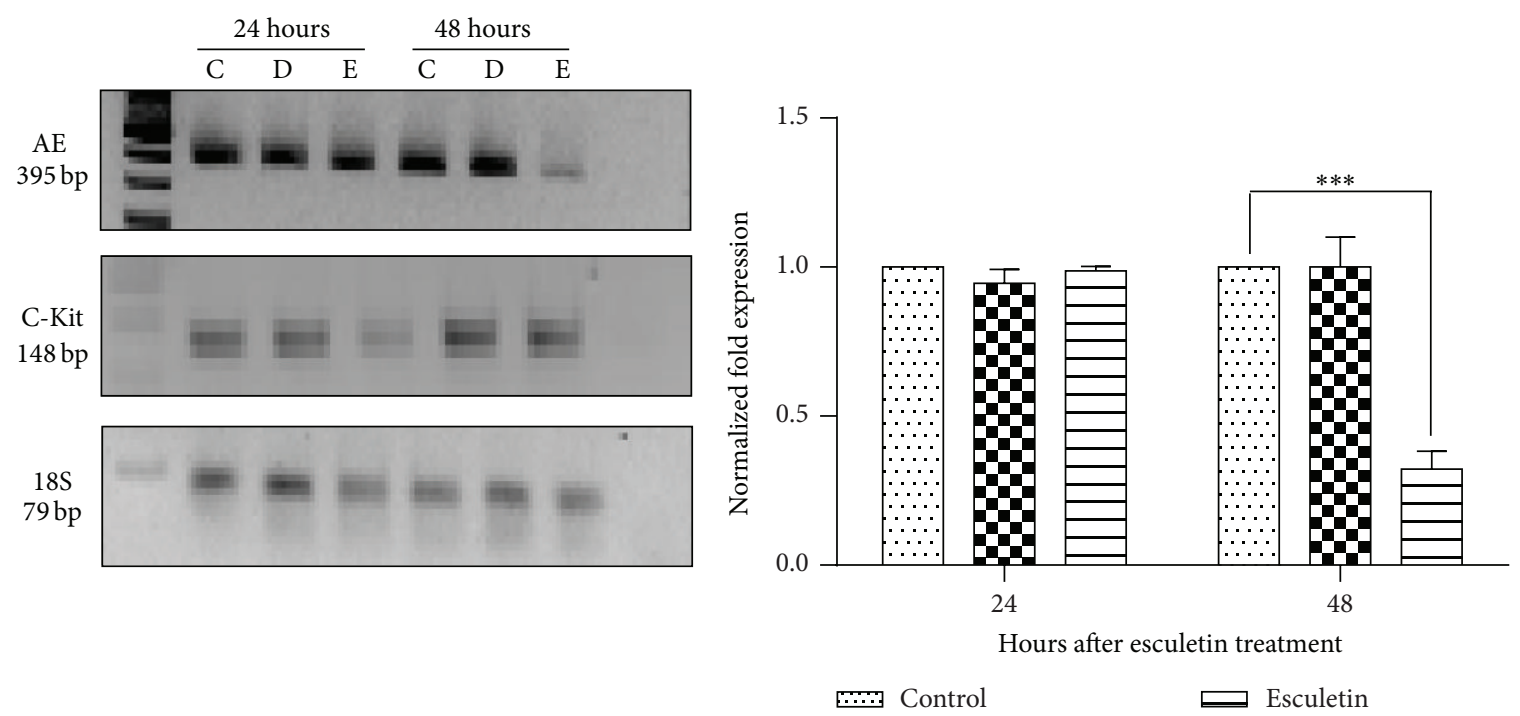

DMSO (a)

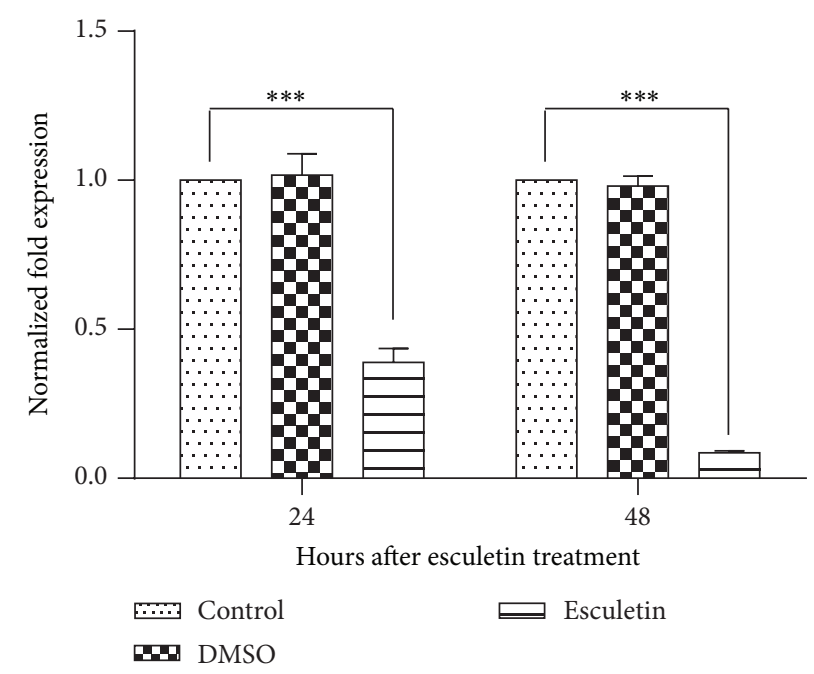

(b)

(c)

FIGURE 2: Esculetin treatment alters the transcript levels of AML1-ETO and C-Kit in Kausmi-1 cells. Kausmi-1 cells were used in these analyses. The results of semiquantitative PCR were for AML1-ETO and C-Kit using cDNA as template; Kausmi-1 cells were incubated with $100 \mu \mathrm{M}$ esculetin (E) and DMSO (D) (vehicle control) along with control untreated (C) for 24 hours and 48 hours (a); results of qPCR for AML1-ETO (b) and C-Kit (c) in esculetin treated and control Kausmi-1 cells are shown. The duration of treatment is indicated on top of the lanes (I). 18SrRNA was used as an internal control for semiquantitative PCR; for qPCR 18SrRNA and GUSB were used for normalization. The data represents mean $\pm \mathrm{SD} .{ }^{* * *} \mathrm{P}<0.05$ compared to control.

and C-Kit activating mutation N822K (unpublished data). Translocations and mutations compliment the proliferation activity, progression of malignancy, and aggressiveness of the cancer. Multiple mutations in $\mathrm{C}$-Kit gene along with AML1-ETO oncogene are the most common pair of genetic anomalies found in $t(8 ; 21)$ type of AML. Thus, there is an exigency to identify a molecule which can simultaneously target both these genes for the efficacious treatment and preponderant outcomes $[1,34]$.

3.1. Effect of Esculetin on Expression of Chimeric Proteins. Oridonin, a tetracycline diterpenoid compound, was recently shown to degrade the chimeric AML1-ETO protein in a caspase-3 dependent manner, although oridonin has no effect on the transcription level of AML1-ETO [21]. We, therefore, examined the effect of esculetin on the expression of not only chimeric AML1-ETO protein but also C-Kit, a tyrosine kinase, using Kasumi-1 cell line as a model system. Kasumi-1 cells were treated with $100 \mu \mathrm{M}$ esculetin along with controls and protein was resolved on $8 \%$ SDS PAGE and probed with anti-AE and anti-C-Kit antibody. As shown in Figure 1(a), the expression of $\mathrm{AE}$ protein decreased as a function of time of esculetin treatment. Interestingly, we also observed a parallel decrease in the expression of C-Kit in esculetin treated 

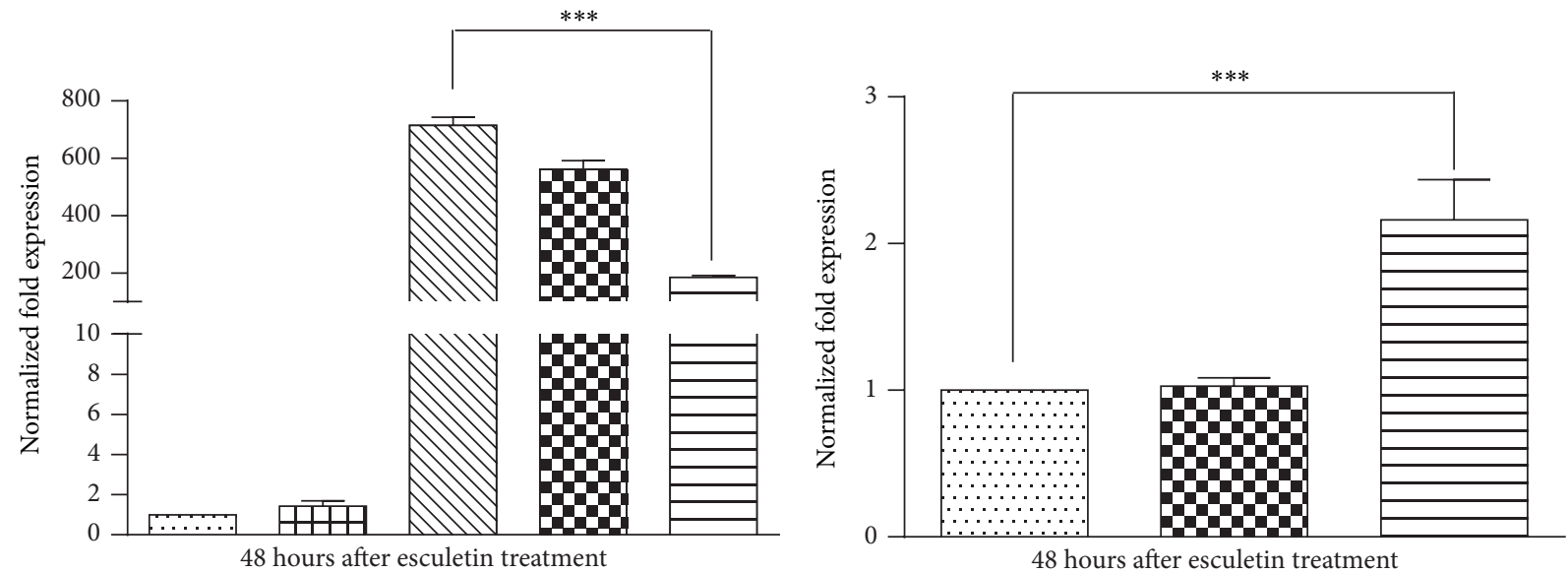

48 hours after esculetin treatment

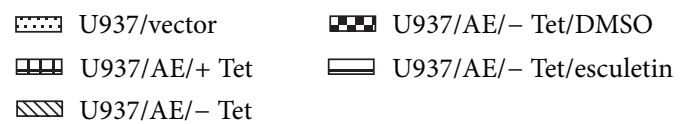

….. Control

DMSO

(a)

(b)

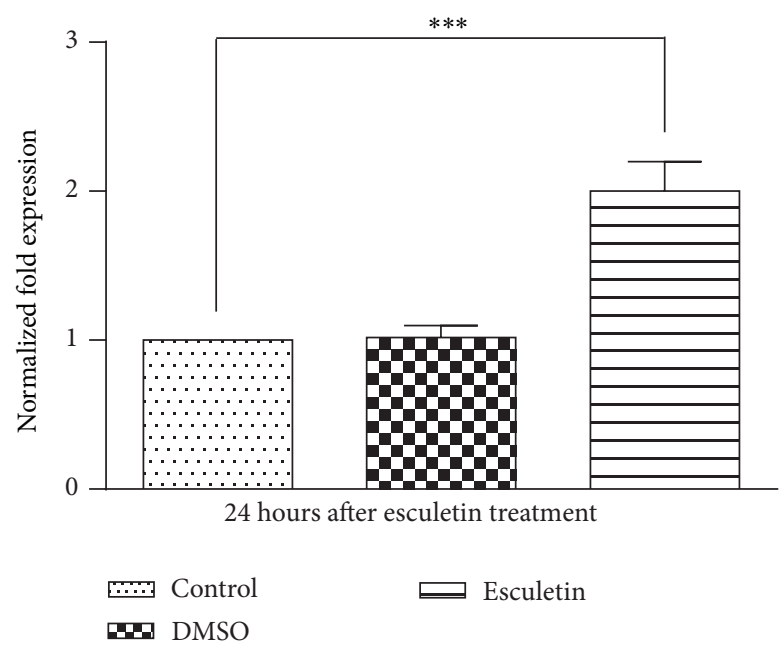

(c)

FIGURE 3: Effect of esculetin on the expression of AML1-ETO in transfected U937 cells (a). AML1-ETO expression was analyzed in untreated U937 cells/vector, U937/AE/+ Tet/, U937/AE/- Tet/, U937/AE/- Tet/D, and U937/AE/- Tet/E 48 hours after esculetin treatment. AML1-ETO (AE), tetracycline (Tet), DMSO (D), and esculetin (E). Esculetin affects the expression of LAT1 and RUNX3 genes, targets of AML1 (a, b, and c). Kasumi-1 cells were treated with $100 \mu \mathrm{M}$ of esculetin for 48 hours for LAT1 and 24 hours for RUNX3; mRNA expression level for both the genes was determined using quantitative real time PCR. 18SrRNA and GUSB genes were used for normalization. An increase in the expression of $L A T 1$ and RUNX3 gene suggests increased expression of AML-1 targets. The data represents mean $\pm \mathrm{SD},{ }^{* * *} P<0.05$ compared to control.

Kasumi-1 cells (Figure 1(b)). Importantly, esculetin treatment of cells did not result in the generation of cleaved form (delta form) of AML1-ETO chimeric protein. This is in contrast to the observations reported earlier wherein oridonin treatment of Kasumi-1 cell line was shown to cleave the chimeric AML1ETO protein [21]. This impelled us to examine whether this decrease in the level of protein is due to modulation of transcription of these genes.

3.2. Effect of Esculetin on Expression of Chimeric Gene. To examine the effect of esculetin on transcription of AML1-ETO and $C$-Kit gene, Kasumi-1 cells were treated with $100 \mu \mathrm{M}$ of esculetin, RNA was isolated, and expression was analysed by semiquantitative RT-PCR. As shown in Figure 2(a), esculetin treatment for 48 hours downregulates the expression of AML1-ETO gene as compared to the DMSO vehicle control. Importantly, the expression of mutated C-Kit gene was also found to be substantially downregulated at 24 hours of esculetin treatment and it reached below the detectable limits by 48 hours. This observation was further substantiated by quantitative RT-PCR analysis, wherein the expression of AML1-ETO and C-Kit was compared in Kasumi-1 cells after 24 and 48 hours of esculetin treatment to untreated controls (Figures 2(b) and 2(c)). C-Kit expression was downregulated 


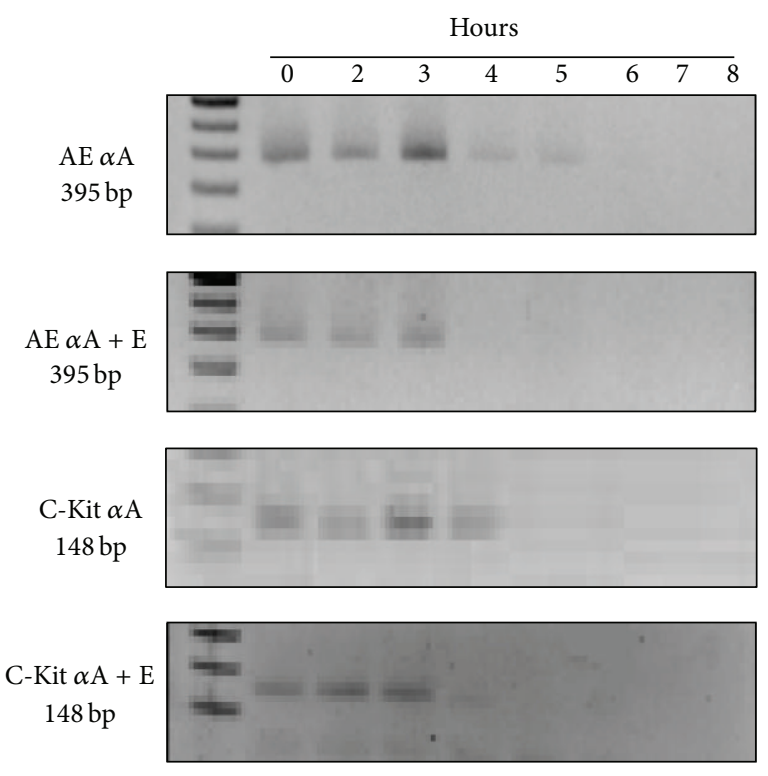

(a)

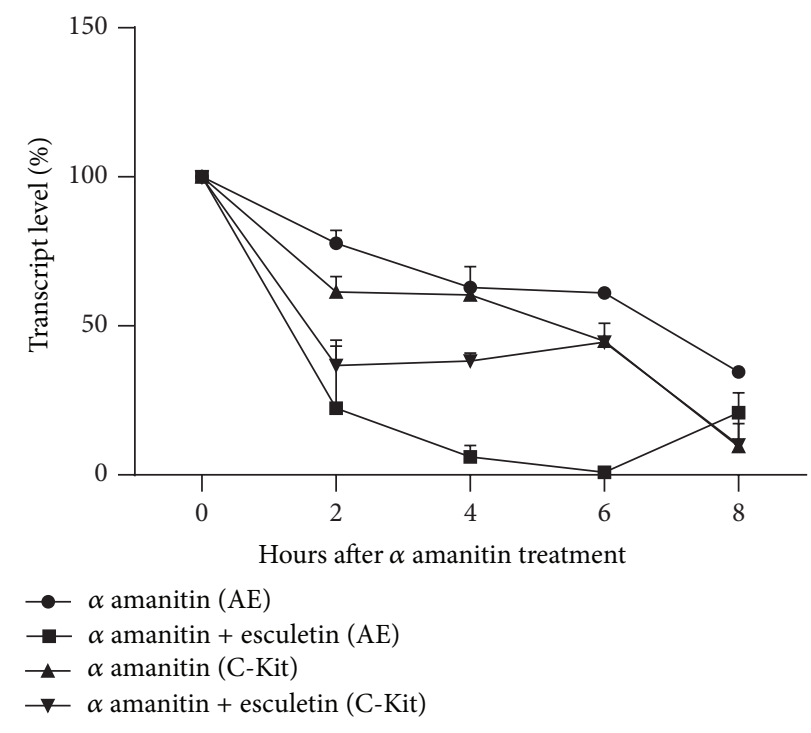

(b)

Figure 4: Esculetin treatment reduces half-life of AML1-ETO and C-Kit mRNA. The levels of mRNA for AML1-ETO (AE) and C-Kit were quantified by semiquantitative RT-PCR (a) and qPCR (b) in esculetin (E) treated and untreated Kausmi-1 cells incubated with $\alpha$ amanitin $(\alpha \mathrm{A})$ for 2-8 hours.

more than 2-fold within 24 hours of esculetin treatment, while at 48 hours, the expression was almost imperceptible. Similarly, esculetin resulted in about $60 \%$ inhibition of AML1ETO expression at 48 hours. $18 S$ rRNA and glucuronidase beta (GUSB) gene expression was used to normalize the data. These observations suggested that esculetin mediated decrease in the protein expression of $\mathrm{AE}$ as well as C-Kit could possibly be due to decreased transcript rather than the cleavage of the protein. We also compared the expression of chimeric gene in U937AE cells stably transfected with recombinant plasmid carrying AML1-ETO c-DNA (please see Section 2 for details). Esculetin treatment substantially downregulated the levels of ectopically expressed AML1ETO (Figure 3(a)). Since the chimeric gene in these cells was not under the regulation of natural promoter, this observation also suggested that esculetin may not be affecting the transcription initiation; rather its effect may be posttranscriptional. Further, we observed that the decrease in AML1-ETO levels in Kasumi-1 cells treated with esculetin was accompanied by an increase in the expression of AML-1 target genes, namely, LAT1 (Figure 3(b)) and RUNX3 (Figure 3(c)). The level of RUNX-1, mRNA was however not significantly altered in the presence of esculetin. Based on these observations, we propose that, under reduced levels of the chimeric protein, which acts as a repressor, the normal RUNX-1 protein is now able to activate the expression of its target genes. These results also suggested that the effect of esculetin on C-Kit and AML1-ETO transcript is specific and not due to general transcription inhibition and/or degradation of mRNA. To further analyse if this decrease in the transcript level is due to decrease in transcription or due to increased degradation, we determined the half-life of the mRNA for C-Kit and AML1-ETO. Transcription of mRNA was inhibited in esculetin treated and untreated Kasumi- 1 cells by incubating the Kasumi- 1 cells in medium containing $50 \mu \mathrm{M}$ of $\alpha$-amanitin, an intense inhibitor of RNA polymerase II [35]. Total RNA was isolated from these cells at defined time interims and subjected to qPCR as described in Section 2. RNA concentration was estimated utilizing standard graph generated from cloned cDNA of AML1-ETO and C-Kit as described in Section 2. Interestingly, we observed a substantial decrease in the half-life of mRNA both for AML1-ETO and C-Kit (see Figure 4(a)). In the absence of esculetin, the half-life of AML1-ETO mRNA was determined to be 7 hours, while it diminished to 1.5 hours in the presence of esculetin. Similarly, the half-life of C-Kit mRNA was reduced from 5 hours in untreated control cells to 2 hours when cells were treated with esculetin (see Figure 4(b)).

\section{Conclusion}

Our experiments clearly establish that esculetin reduces the levels of C-Kit as well as chimeric gene AML1-ETO by altering the half-life of mRNA. Although, at present the mechanism by which esculetin reduces the half-life of mRNA is not known, future studies are being directed to check the effect of esculetin in posttranscriptional modifications and/or splicing of precursor RNA. Earlier, the siRNA mediated "knockdown" of the mRNA of AML-ETO was shown to result in growth arrest, granulocytic differentiation, and modulation of expression of genes involved in cell-cycle control and differentiation $[36,37]$. To the best of our knowledge, this is 
the first report wherein a natural coumarin has been shown to regulate the expression of oncogenes, namely, AML1-ETO and $C$-Kit, at the posttranscriptional level. It appears that the impact of esculetin on the levels of both AML1-ETO and CKit may be a consequence of an indirect mechanism. It is possible that such a mechanism may involve the downregulation of another transcription factor. We, therefore, propose that use of esculetin may provide an important therapeutic approach for $\mathrm{t}(8 ; 21)$ positive leukemia.

\section{Conflict of Interests}

The authors declare that they have no conflict of interests.

\section{Authors' Contribution}

Daman Saluja and Sharad Sawney conceived the study and participated in its design and coordination. Sharad Sawney and Rashi Arora carried out the experiments and Kamal K. Aggarwal participated in the design of the study. Sharad Sawney and Daman Saluja drafted the paper. All authors read and approved the final paper.

\section{Acknowledgments}

Sharad Sawney acknowledges Senior Research Fellowship provided by Indian Council of Medical Research (ICMR), New Delhi. Rashi Arora acknowledges Senior Research Fellowship provided by University Grant Commision. Authors acknowledge the benevolence of Professor Olef Hendrich Newcastle University, Northern Institute for Cancer Research, UK, and Professor Dong-Er Zhang Department of Pathology and Division of Biological Sciences, Moores Cancer Center University of California, San Diego, USA, for sharing the cell lines.

\section{References}

[1] A. V. Rulina, P. V. Spirin, and V. S. Prassolov, "Activated leukemic oncogenes AML1-ETO and c-kit: role in development of acute myeloid leukemia and current approaches for their inhibition," Biochemistry, vol. 75, no. 13, pp. 1650-1666, 2010.

[2] L. F. Peterson and D.-E. Zhang, "The 8;21 translocation in leukemogenesis," Oncogene, vol. 23, no. 24, pp. 4255-4262, 2004.

[3] L. M. Kelly and D. G. Gilliland, "Genetics of myeloid leukemias," Annual Review of Genomics and Human Genetics, vol. 3, pp. 179198, 2002.

[4] N. A. Speck and D. G. Gilliland, "Core-binding factors in haematopoiesis and leukaemia," Nature Reviews Cancer, vol. 2, no. 7, pp. 502-513, 2002.

[5] A. Lasa, M. T. Carricondo, M. J. Carnicer, G. Perea, A. Aventín, and J. F. Nomdedeu, "A new D816 c-KIT gene mutation in refractory AML1-ETO leukemia," Haematologica, vol. 91, no. 9, pp. 1283-1284, 2006.

[6] D. C. Arthur and C. D. Bloomfield, "Partial deletion of the long arm of chromosome 16 and bone marrow eosinophilia in acute nonlymphocytic leukemia: a new association," Blood, vol. 61, no. 5, pp. 994-998, 1983.
[7] D. G. Gilliland, "Molecular genetics of human leukemia," Leukemia, vol. 12, supplement 1, pp. S7-S12, 1998.

[8] C. Perry, A. Eldor, and H. Soreq, "Runx1/AML1 in leukemia: disrupted association with diverse protein partners," Leukemia Research, vol. 26, no. 3, pp. 221-228, 2002.

[9] B. A. Hug and M. A. Lazar, "ETO interacting proteins," Oncogene, vol. 23, no. 24, pp. 4270-4274, 2004.

[10] K. E. Elagib and A. N. Goldfarb, "Oncogenic pathways of AML1ETO in acute myeloid leukemia: multifaceted manipulation of marrow maturation," Cancer Letters, vol. 251, no. 2, pp. 179-186, 2007.

[11] S. Minucci, M. Maccarana, M. Cioce et al., "Oligomerization of RAR and AML1 transcription factors as a novel mechanism of oncogenic activation," Molecular Cell, vol. 5, no. 5, pp. 811-820, 2000.

[12] G. Frosina, "Overexpression of enzymes that repair endogenous damage to DNA," European Journal of Biochemistry, vol. 267, no. 8, pp. 2135-2149, 2000.

[13] B. Niebuhr, M. Fischer, M. Täger, J. Cammenga, and C. Stocking, "Gatekeeper function of the RUNX1 transcription factor in acute leukemia," Blood Cells, Molecules, and Diseases, vol. 40, no. 2, pp. 211-218, 2008.

[14] B. J. Longley Jr., D. D. Metcalfe, M. Tharp et al., "Activating and dominant inactivating c-KIT catalytic domain mutations in distinct clinical forms of human mastocytosis," Proceedings of the National Academy of Sciences of the United States of America, vol. 96, no. 4, pp. 1609-1614, 1999.

[15] F. Zaker, M. Mohammadzadeh, and M. Mohammadi, "Detection of KIT and FLT3 mutations in acute myeloid leukemia with different subtypes," Archives of Iranian Medicine, vol. 13, no. 1, pp. 21-25, 2010.

[16] S. H. Park, H.-S. Chi, S.-K. Min, B. G. Park, S. Jang, and C.-J. Park, "Prognostic impact of c-KIT mutations in core binding factor acute myeloid leukemia," Leukemia Research, vol. 35, no. 10, pp. 1376-1383, 2011.

[17] J. M. Rowe and M. S. Tallman, "How I treat acute myeloid leukemia," Blood, vol. 116, no. 17, pp. 3147-3156, 2010.

[18] D. Osman, V. Gobert, F. Ponthan, O. Heidenreich, M. Haenlin, and L. Waltzer, "A Drosophila model identifies calpains as modulators of the human leukemogenic fusion protein AML1ETO," Proceedings of the National Academy of Sciences of the United States of America, vol. 106, no. 29, pp. 12043-12048, 2009.

[19] W. Jin, K. Wu, Y. Z. Li et al., "AML1-ETO targets and suppresses cathepsin G, a serine protease, which is able to degrade AML1ETO in $\mathrm{t}(8 ; 21)$ acute myeloid leukemia," Oncogene, vol. 32, no. 15, pp. 1978-1987, 2013.

[20] S. M. Corsello, G. Roti, K. N. Ross et al., "Identification of AML1ETO modulators by chemical genomics," Blood, vol. 113, no. 24, pp. 6193-6205, 2009.

[21] G.-B. Zhou, H. Kang, L. Wang et al., "Oridonin, a diterpenoid extracted from medicinal herbs, targets AML1-ETO fusion protein and shows potent antitumor activity with low adverse effects on $\mathrm{t}(8 ; 21)$ leukemia in vitro and in vivo," Blood, vol. 109, no. 8, pp. 3441-3450, 2007.

[22] W. S. Chang, C. C. Lin, S. C. Chuang, and H. C. Chiang, "Superoxide anion scavenging effect of coumarins," The American Journal of Chinese Medicine, vol. 24, no. 1, pp. 11-17, 1996.

[23] J.-M. Yue, J. Xu, Y. Zhao, H.-D. Sun, and Z.-W. Lin, "Chemical components from Ceratostigma willmottianum," Journal of Natural Products, vol. 60, no. 10, pp. 1031-1033, 1997. 
[24] K. Matsunaga, N. Yoshimi, Y. Yamada et al., "Inhibitory effects of Nabumetone, a cyclooxygenase-2 inhibitor, and esculetin, a lipoxygenase inhibitor, on $\mathrm{N}$-methyl-N-nitrosourea-induced mammary carcinogenesis in rats," Japanese Journal of Cancer Research, vol. 89, no. 5, pp. 496-501, 1998.

[25] M. Noguchi, M. Earashi, M. Minami, I. Miyazaki, M. Tanaka, and T. Sasaki, "Effects of piroxicam and esculetin on the MDA-MB-231 human breast cancer cell line," Prostaglandins Leukotrienes and Essential Fatty Acids, vol. 53, no. 5, pp. 325329, 1995.

[26] C.-J. Wang, Y.-J. Hsieh, C.-Y. Chu, Y.-L. Lin, and T.-H. Tseng, "Inhibition of cell cycle progression in human leukemia HL-60 cells by esculetin," Cancer Letters, vol. 183, no. 2, pp. 163-168, 2002.

[27] S. A. Burel, N. Harakawa, L. Zhou, T. Pabst, D. G. Tenen, and D.E. Zhang, "Dichotomy of AML1-ETO functions: growth arrest versus block of differentiation," Molecular and Cellular Biology, vol. 21, no. 16, pp. 5577-5590, 2001.

[28] K. V. Prasanth, M. Camiolo, G. Chan et al., "Nuclear organization and dynamics of 7SK RNA in regulating gene expression," Molecular Biology of the Cell, vol. 21, no. 23, pp. 4184-4196, 2010.

[29] I. S. Lossos, D. K. Czerwinski, M. A. Wechser, and R. Levy, "Optimization of quantitative real-time RT-PCR parameters for the study of lymphoid malignancies," Leukemia, vol. 17, no. 4, pp. 789-795, 2003.

[30] F. Rosenbauer, S. Koschmieder, U. Steidl, and D. G. Tenen, "Effect of transcription-factor concentrations on leukemic stem cells," Blood, vol. 106, no. 5, pp. 1519-1524, 2005.

[31] H. Reikvam, K. J. Hatfield, A. O. Kittang, R. Hovland, and O. Bruserud, "Acute myeloid leukemia with the $\mathrm{t}(8 ; 21)$ translocation: clinical consequences and biological implications," Journal of Biomedicine and Biotechnology, vol. 2011, Article ID 104631, 2011.

[32] A. M. S. Müller, J. Duque, J. A. Shizuru, and M. Lübbert, "Complementing mutations in core binding factor leukemias: from mouse models to clinical applications," Oncogene, vol. 27, no. 44, pp. 5759-5773, 2008.

[33] C. Park, C.-Y. Jin, G.-Y. Kim et al., "Induction of apoptosis by esculetin in human leukemia U937 cells through activation of JNK and ERK," Toxicology and Applied Pharmacology, vol. 227, no. 2, pp. 219-228, 2008.

[34] L. K. Ashman and R. Griffith, "Therapeutic targeting of c-KIT in cancer," Expert Opinion on Investigational Drugs, vol. 22, no. 1, pp. 103-115, 2013.

[35] T. J. Lindell, F. Weinberg, P. W. Morris, R. G. Roeder, and W. J. Rutter, "Specific inhibition of nuclear RNA polymerase II by $\alpha$-amanitin," Science, vol. 170, no. 3956, pp. 447-449, 1970.

[36] O. Heidenreich, J. Krauter, H. Riehle et al., "AML1/MTG8 oncogene suppression by small interfering RNAs supports myeloid differentiation of $\mathrm{t}(8 ; 21)$-positive leukemic cells," Blood, vol. 101, no. 8, pp. 3157-3163, 2003.

[37] J. Dunne, C. Cullmann, M. Ritter et al., "siRNA-mediated AML1/MTG8 depletion affects differentiation and proliferation-associated gene expression in $\mathrm{t}(8 ; 21)$-positive cell lines and primary AML blasts," Oncogene, vol. 25, no. 45, pp. 6067-6078, 2006. 


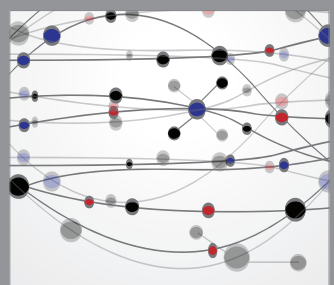

The Scientific World Journal
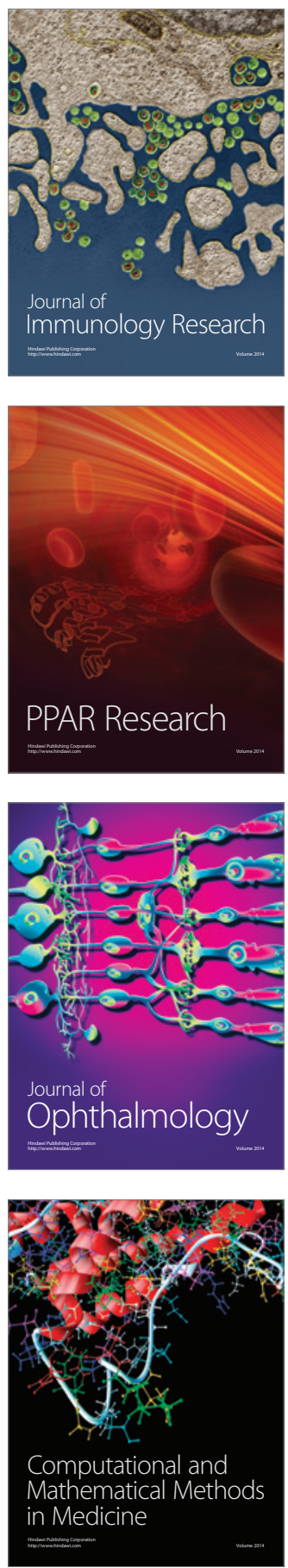

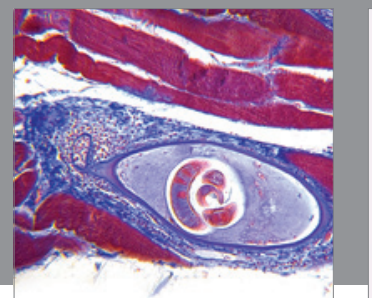

Gastroenterology

Research and Practice
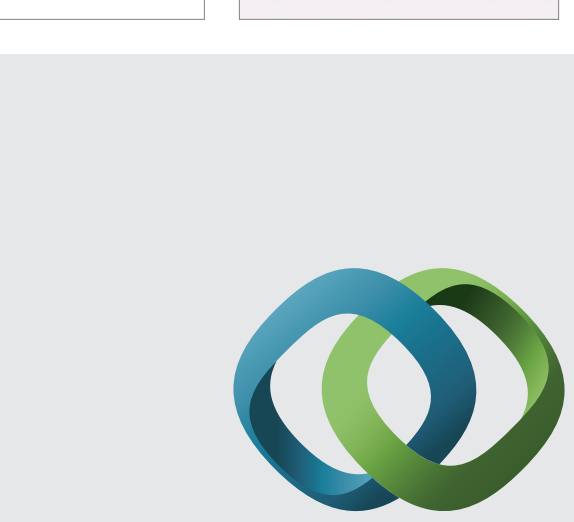

\section{Hindawi}

Submit your manuscripts at

http://www.hindawi.com
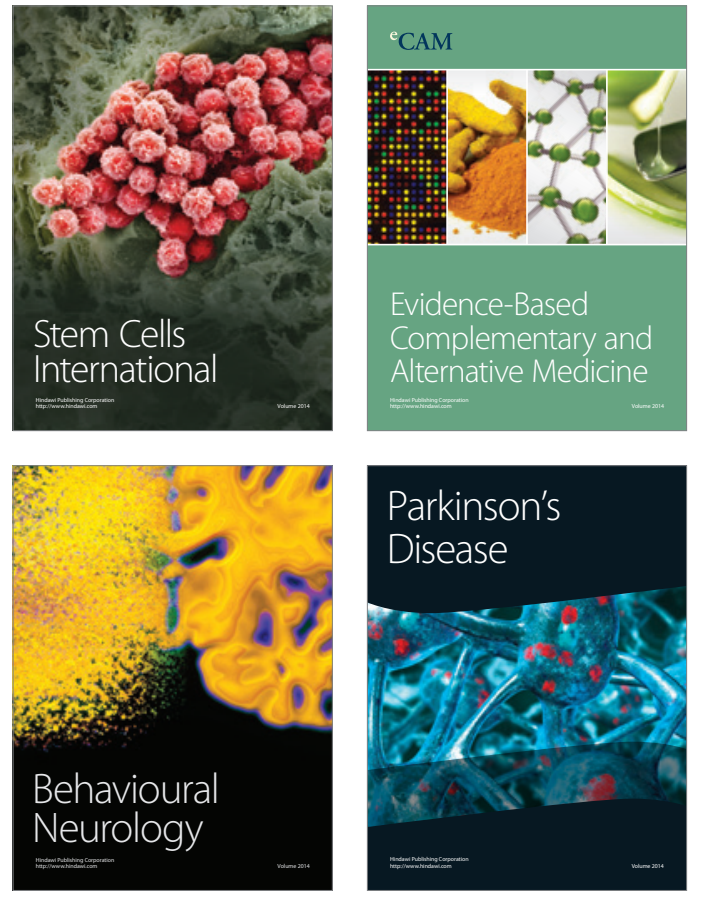
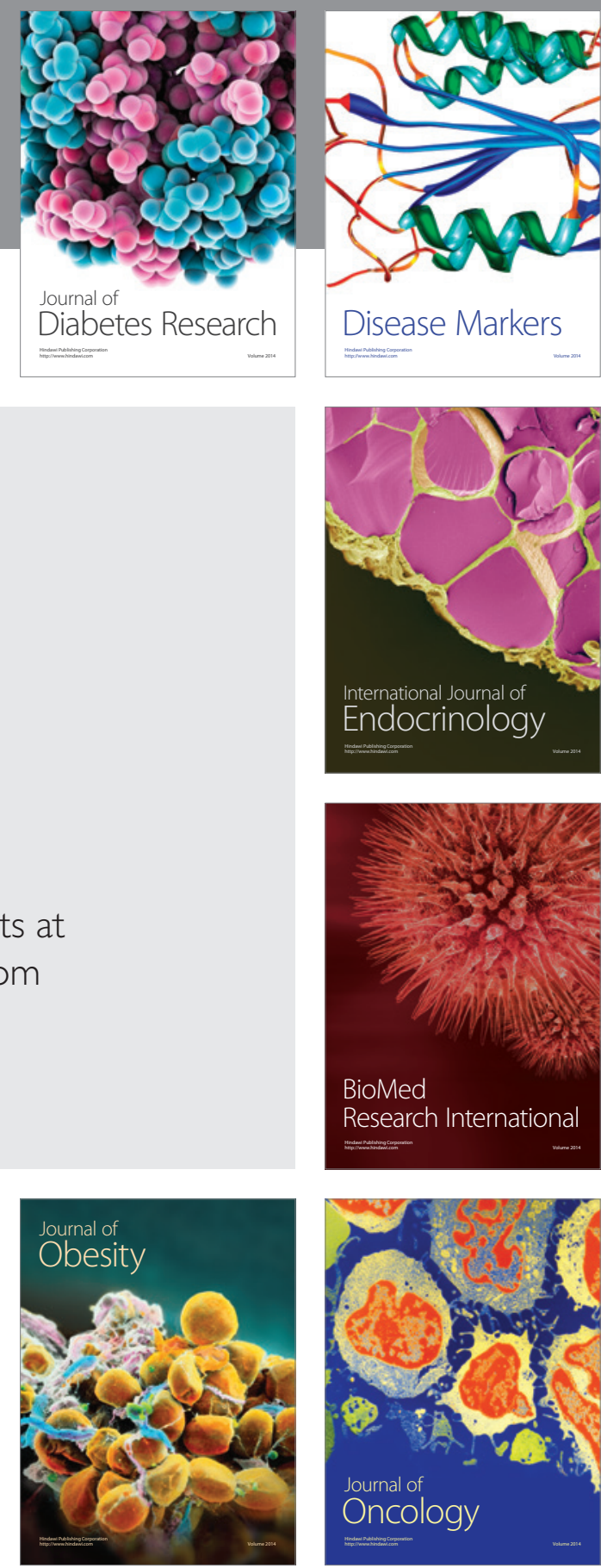

Disease Markers
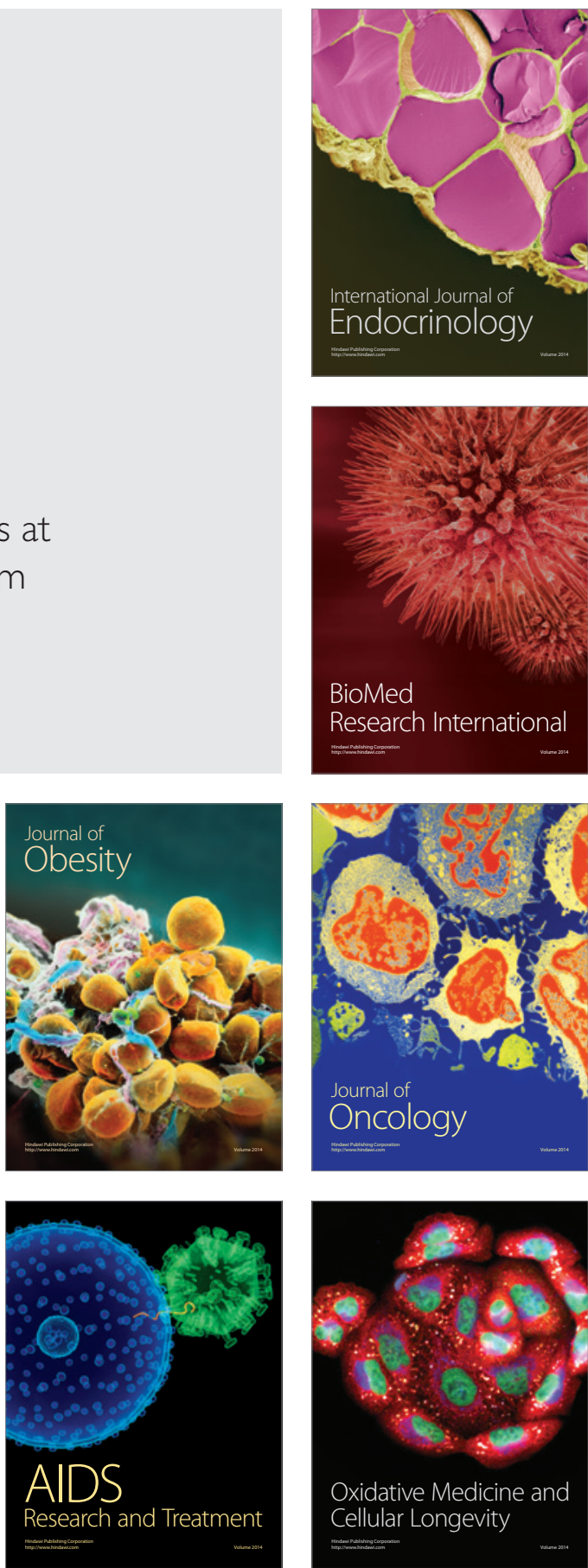